Original research

\title{
The effects of cold water immersion with different dosages (duration and temperature variations) on heart rate variability post-exercise recovery: A randomized controlled trial
}

\author{
Aline C. Almeida ${ }^{\mathrm{a}, *}$, Aryane F. Machado ${ }^{\mathrm{b}}$, Maíra C. Albuquerque ${ }^{\mathrm{b}}$, Lara M. Netto ${ }^{\mathrm{b}}$, \\ Franciele M. Vanderlei ${ }^{\mathrm{b}}$, Luiz Carlos M. Vanderlei ${ }^{\mathrm{b}}$, Jayme Netto Junior ${ }^{\mathrm{b}}$, \\ Carlos M. Pastre ${ }^{b}$ \\ a Univ Federal de São Carlos, Centro de Ciências Biológicas e da Saúde, Departamento de Fisioterapia, Brazil \\ ${ }^{\mathrm{b}}$ Univ Estadual Paulista, Faculdade de Ciências e Tecnologia, Departamento de Fisioterapia, Presidente Prudente, Brazil
}

\section{A R T I C L E I N F O}

\section{Article history:}

Received 7 April 2015

Received in revised form

21 September 2015

Accepted 7 October 2015

Available online 10 November 2015

\section{Keywords:}

Recovery of function

Autonomic nervous system

Cryotherapy

Immersion

\begin{abstract}
A B S T R A C T
Objectives: The aim of the present study was to investigate the effects of cold water immersion during post-exercise recovery, with different durations and temperatures, on heart rate variability indices. Design: Hundred participants performed a protocol of jumps and a Wingate test, and immediately afterwards were immersed in cold water, according to the characteristics of each group (CG: control; G1: $5^{\prime}$ at $9 \pm 1^{\circ} \mathrm{C} ; \mathrm{G} 2: 5^{\prime}$ at $14 \pm 1^{\circ} \mathrm{C} ; \mathrm{G} 3: 15^{\prime}$ at $9 \pm 1^{\circ} \mathrm{C} ; \mathrm{G} 4: 15^{\prime}$ at $14 \pm 1^{\circ} \mathrm{C}$ ).

Methods: Analyses were performed at baseline, during the CWI recuperative technique (TRec) and 20, 30, 40, 50 and 60 min post-exercise. The average HRV indices of all RR-intervals in each analysis period (MeanRR), standard deviation of normal RR-intervals (SDNN), square root of the mean of the sum of the squares of differences between adjacent RR-intervals (RMSSD), spectral components of very low frequency (VLF), low frequency (LF) and high frequency (HF), scatter of points perpendicular to the line of identity of the Poincaré Plot (SD1) and scatter points along the line of identity (SD2) were assessed. Results: Mean RR, VLF and LF presented an anticipated return to baseline values at all the intervention groups, but the same was observed for SDNN and SD2 only in the immersion for 15 min at $14{ }^{\circ} \mathrm{C}$ group (G4). In addition, G4 presented higher values when compared to CG.

Conclusions: These findings demonstrate that if the purpose of the recovery process is restoration of cardiac autonomic modulation, the technique is recommended, specifically for $15 \min$ at $14^{\circ} \mathrm{C}$.
\end{abstract}

C 2015 Sports Medicine Australia. Published by Elsevier Ltd. All rights reserved.

\section{Introduction}

Post-exercise recovery has been extensively investigated in the scientific scenario, ${ }^{22}$ including techniques to accelerate the recovery process after exercise. Furthermore, studies investigating the effects of cold water immersion (CWI), which consists of body segments immersed in water at temperatures below $15^{\circ} \mathrm{C}$, have been encouraging. ${ }^{7}$

Analysis of heart rate variability (HRV) indices as indicators of autonomic nervous system (ANS) activity, ${ }^{2,5,8}$ which performs a significant role in maintaining homeostasis, has been studied. . $^{5,9}$ During exercise, sympathetic activity is increased, while during the

\footnotetext{
* Corresponding author.

E-mail address: alinecastilho@live.com (A.C. Almeida).
}

post-exercise period there is a concomitant progressive parasympathetic reactivation with a sympathetic withdrawal. ${ }^{2}$ The balance between sympathetic and parasympathetic activity reflects autonomic recovery and restoration of cardiovascular homeostasis which is an important component of overall recovery. ${ }^{20}$

Considering that exercise acts as a stressor agent, which causes metabolic and autonomic changes, ${ }^{4,12}$ it is important to understand the possible post-exercise effects of CWI on ANS. Studies ${ }^{2,5,8}$ suggest that CWI results in better ANS activity post-exercise, anticipating the recovery of HRV indices. This can potentially improve the adaptation to training loads or at least reduce stress-related symptoms ${ }^{23}$ leading to an imbalance stress-recovery ${ }^{15}$ and can confer a great cardioprotective effect. ${ }^{20}$

Bastos et al. ${ }^{5}$ observed higher cardiac autonomic modulation on post-exercise recovery after CWI application performed at $11 \pm 2{ }^{\circ} \mathrm{C}$ for $6 \mathrm{~min}$, with higher parasympathetic indices compared to other 
recuperative techniques. The authors propose time and temperature application of this technique based on previous experience. This design is one of the various strategies commonly used. Pastre et al.,22 in a systematic review, evidenced inconsistencies in studies using recovery techniques and attribute this to the variety of applications methods as immersion of members or total body, temperature and time of application used in CWI and the models of physical stress. The lack of the scientific literature refers to the absence of the association between dosages of intervention and the results. Therefore, it seems pertinent to investigate variables such as duration and temperature during CWI application.

The aim of this study was to investigate the effects of CWI on HRV indices as a post-exercise recovery technique, using different durations and temperatures. It was hypothesized that CWI, regardless of the application dosage, would be able to promote better autonomic modulation, thus anticipating the recovery moment. In addition it is believed that the immersion dosage is a decisive factor for optimizing the recovery of HRV indices, and CWI used for long durations and lower temperatures will present the positive effects already mentioned in previous studies.

\section{Methods}

Hundred young healthy male participants (aged 21.73 \pm 2.92 years, height $1.75 \pm 0.06 \mathrm{~m}$, weight $74.22 \pm 10.15 \mathrm{~kg}$, BMI $24.15 \pm 2.99 \mathrm{~kg} \mathrm{~m}^{-2}$ ) were recruited, with a minimum classification requirement of being physically active according to the International Physical Activity Questionnaire (IPAQ). To be included, participants could not be smokers, alcoholics, using drugs that influenced cardiac autonomic activity or have cardiovascular, metabolic or endocrine diseases, as reported in a previous interview. $^{5}$

To determine the sample size a priori knowledge was used, based on Bastos et al. ${ }^{5}$ findings for SDNN, which represents the activity of both ANS branches, indicating the overall behavior of HRV. ${ }^{29}$ A sample size of 18 participants per group was stipulated by a test of hypothesis (two-tail), with $5 \%$ level of significance and $80 \%$ power. The participants were randomized (Microsoft Excel software) and allocated into five groups, one control (CG-passive recovery), and four intervention (G1, G2, G3 and G4).

Following the analysis, there was some data loss due to the time series presenting less than $95 \%$ sinus beats. There was no loss in CG $(n=20)$, one loss in G1 $(n=19)$ and G2 $(n=19)$, two losses in G3 $(n=18)$ and no losses in G4 $(n=20)$.

All procedures were approved by the Research Ethics Committee of the Univ. Estadual Paulista (Protocol 51903/2012) and were performed in a laboratory with an average temperature of $22.0 \pm 2.34{ }^{\circ} \mathrm{C}$ and relative humidity between $40 \%$ and $60 \%$, between 5 and 10 pm to avoid circadian variation. ${ }^{5}$ Participants were advised not to perform vigorous exercise for either $24 \mathrm{~h}$ before and during the study, refrain from drinks and stimulants containing caffeine and any other supplements that might promote recovery. However, it was not carried out based on any monitoring beyond questioning the participants.

In order to capture the RR-intervals parameters, for further analysis of HRV, a chest belt was placed over the precordium region and a heart rate receiver (Polar RS800CX, Polar Electro-Oy, Finland) on the wrist. ${ }^{12}$ During capture, the participants remained at rest in a sitting position breathing spontaneously.

The exercise protocol consisted of a jumping protocol and a short-duration maximal cycling test. The jumping protocol was composed of 10 sets of 10 maximal vertical jumps with a $1 \mathrm{~min}$ break between sets. Prior to starting the exercise, a maximal vertical jump was performed with a chalk mark made by the fingertips at the highest point of the jump. This mark was subsequently used as a target height that the participant attempted to maintain for each jump. On landing, participants were instructed to position their hands on their hips and adopt a knee joint angle of approximately $90^{\circ}$ to avoid compensation. ${ }^{28}$

Immediately after, participants performed the short-duration maximal cycling test, consisting of a Wingate Test, on a cycloergometer (Biotec 2100-Cefise, Brazil), which triggers cardiac autonomic derangement. ${ }^{18}$ The participants performed a 5 min warm-up, consisting of pedaling with a load of $1.0 \mathrm{~kg}$ at $60-90 \mathrm{rpm}$ and sprints in the 2 nd and 4 th min, followed by maximum pedaling for $30 \mathrm{~s}$ with a load of $0.075 \mathrm{kp} \mathrm{kg}^{-1}$ of participant's body mass. ${ }^{18}$ These stress protocols were considered to cause exhaustion in both musculoskeletal and metabolic systems.

Immediately after the stress protocol, an interval of $1 \mathrm{~min}$ was allowed for the transition to the recovery intervention and participants from the intervention groups were immersed up to the height of anterior-superior iliac spine, whilst remaining seated. The duration and water temperature of CWI were established in accordance with the characteristics of each group. G1 remained immersed for $5 \mathrm{~min}$ at $9 \pm 1{ }^{\circ} \mathrm{C} ; \mathrm{G} 2: 5 \mathrm{~min}$ at $14 \pm 1^{\circ} \mathrm{C}$; G3:15 min at $9 \pm 1{ }^{\circ} \mathrm{C}$ and G4: $15 \mathrm{~min}$ at $14 \pm 1^{\circ} \mathrm{C}$. The immersion duration was controlled by a timer and the temperature was controlled by putting ice and stirring the water, and constantly evaluated by a thermometer with $\pm 0.3^{\circ} \mathrm{C}$ accuracy. CG performed passive recovery seated for $15 \mathrm{~min}$. These durations and temperatures of immersion were established in accordance with the most commonly used strategies in the literature $\mathrm{e}^{7,30}$ and those that allowed a more comprehensive assessment of CWI effects. Participants were not informed either which group they belonged to regarding intervention duration and temperature or which intervention was considered therapeutic. ${ }^{11}$ After the recovery intervention, participants remained seated. The total recovery time was $75 \mathrm{~min}$ including the time the participant remained immersed.

HRV analysis was performed from RR-intervals series at a sampling rate of $1000 \mathrm{~Hz}$ analyzed using the software Polar Pro Trainer 5.0. Digital filtering was supplemented by manual filtering in order to remove the artifacts, ectopic and premature beats, and only series with more than $95 \%$ sinus beats were included. ${ }^{12}$

The extraction of each cardiac period (RR-interval) was analyzed using Kubios HRV Analysis Software 2.0 for Windows software (The Biomedical Signal Analysis Group, Department of Applied Physics, University of Kuopio, Finland), and a fixed number of 256 consecutive RR-intervals ${ }^{12}$ was considered at baseline $(20 \mathrm{~min}$ with the participants at rest, of which the final $256 \mathrm{RR}$-intervals were selected), during the recuperative technique (TRec) (for intervention groups, the final 256 RR-intervals for 5 min for G1 and G2, for $15 \mathrm{~min}$ for G3 and G4, and for 5 or $15 \mathrm{~min}$, for subsequent comparison with the appropriate intervention groups) and at 20, 30, 40, 50 and $60 \mathrm{~min}$ after exercise (128 RR-intervals before and after each time).

For analysis in the time domain (TD), Mean RR (average of all RR-intervals in each analysis period), RMSSD (square root of the average squared differences between adjacent normal RRintervals) and SDNN (standard deviation of normal RR-intervals) indices were analyzed ${ }^{26,29}$ expressed in milliseconds.

In the frequency domain (FD) the spectral components of very low frequency (VLF) $(0.00-0.04 \mathrm{~Hz})$; low frequency (LF) $(0.04-0.15 \mathrm{~Hz})$ and high frequency $(\mathrm{HF})(0.15-0.4 \mathrm{~Hz})^{29}$ were utilized, expressed in $\mathrm{ms}^{2}$, calculated using Fast Fourier Transform. ${ }^{12}$ VLF assessed from short-term recordings, although tedious measure, was considered here for analysis.

For the quantitative analysis of the Poincaré plot (PP), SD1 (scatter of points perpendicular to the line of identity) and SD2 (scatter points along the line of identity) were utilized. ${ }^{16}$

To analyze the sample characteristics considering anthropometric and age data the descriptive statistical method was used and 
the results were presented as mean values and standard deviations. Data normality was assessed (Kolmogorov-Smirnov test), considered normal and therefore the parametric analysis was used. Analysis of one-way variance (ANOVA) was performed, followed by the Tukey's test.

Sphericity of the data of HRV was tested by Mauchly's test. In case of violation of the sphericity assumption, the GreenhouseGeisser corrections were used. Data were analyzed using Repeated Measures Analysis of Variance which provide information of time and group (Bonferroni's test was performed when required). The comparison between the moments was compared to baseline values for all groups (Baseline vs TRec, 20, 30, 40, 50 and 60). The significance level was set at $p<0.05$.

\section{Results}

Anthropometric characteristics and age are presented in Table 1. No significant differences were observed between groups in any variables.

For the TD (Fig. 1), FD (Fig. 2) and PP (Fig. 3) a better recovery was observed, favoring the CWI interventions $(p<0.05)$. For MeanRR (Fig. 1a), VLF (Fig. 2a) and LF (Fig. 2b), there was an earlier recovery between 10 and $20 \mathrm{~min}$ for the CWI groups compared to CG. For SDNN (Fig. 1b) and SD2 (Fig. 3b), G4 presented an earlier recovery (10 min) compared to the other groups. For RMSSD (Fig. 1c), HF (Fig. 2c) and SD1 (Fig. 3a) there was no difference in recovery time for any groups.

In the groups comparison, G4 presented statistically greater $(p<0.05)$ results compared to CG in most indices evaluated between 40 and 60 min post-exercise, with the exception of VLF, which was statistically higher in $G 1(p<0.05)$ at $60 \mathrm{~min}$ postexercise.

In the relationship between time and intervention, there was a significant interaction for MeanRR $(p<0.001)$, SDNN $(p=0.001)$, RMSSD $(p=0.003), \operatorname{VLF}(p=0.025)$, SD1 $(p=0.014)$ and SD2 $(p=0.045)$.

\section{Discussion}

When considering the return to baseline values, 60 min was sufficient in any situation, regardless of the use or not of CWI for recovery of $\mathrm{HRV}$ indices and in overall variability there was a return to baseline values at between 10 and 20 min when CWI was applied. The principal findings were related to the CWI for $15 \mathrm{~min}$ at $14^{\circ} \mathrm{C}$.

The analysis performed during TRec demonstrated similar behavior for all groups. CWI performed at any duration or temperature did not influence the behavior of the ANS, when compared to passive recovery, and all indices presented a significant decrease when compared to baseline, reflected by the autonomic derangement caused by the exercise protocol.

Studies comparing the effects of CWI with other recuperative techniques have found better results during the TRec. ${ }^{3,8}$ The discrepancy between the findings could be related to the stress model used. This study used a supramaximal exercise model, ${ }^{13,18}$ which may have triggered an intense autonomic derangement in an attempt to meet the metabolic needs. ${ }^{17}$ However, a gradual increase in HRV indices presented $20 \mathrm{~min}$ into the recuperative period demonstrated an attempt to return to baseline values, and 60 min time was sufficient for physically active subjects to receive a new stimulus from a cardiac autonomic point of view.

The application of CWI, principally performed for $15 \mathrm{~min}$ at $14^{\circ} \mathrm{C}$, presented the greatest effect on the sympathetic branch of ANS, so as to reduce the modulation in the subsequent moments of immersion. Such behavior was evidenced in the indices that represent the action of both branches, which clearly demonstrated better recovery in all groups that performed CWI for the MeanRR, VLF and LF indices and only in G4 for the SDNN and SD2 indices, which demonstrated an anticipated time of return to baseline values.

However, there were no differences in the recovery times between groups for the indices that represent parasympathetic modulation. Although, when comparing the groups, better results were found for the immersion performed for $15 \mathrm{~min}$ at $14^{\circ} \mathrm{C} \mathrm{com}$ pared to CG in the closing moments of recovery. In the present study we have not investigated parasympathetic reactivation from $\mathrm{CWI}$ action as suggested by Buchheit et al. ${ }^{8}$ Regardless of occurrence of this phenomenon, there is no significant increase in parasympathetic tone in the first analysis intervals. There is, however, a significant rebound after 50 min post-exercise on RMSSD, HF and SD1, referring to the parasympathetic modulation for groups at 15 min of immersion. One hypothesis to explain these results may be related to the duration of dosing that reflects later its impact on the system.

Some studies ${ }^{2,7,8}$ have indicated the occurrence of a cascade of responses promoted by the hemodynamic system after CWI, including flow redirection from the periphery to the central regions of the chest, increased venous return and cardiac output, contributing to higher cardiac autonomic modulation, 2,8,25 which can be reflected, as in the findings of the present study, by anticipated values to baseline in the global indices.

The hypotheses for the described outcomes may be related to two main factors. Although an immediate response was not observed during the TRec, there was a response in baroreceptors stimulus and a temperature reduction through thermoregulation in the subsequent moments. Baroreceptors are sensitive to changes in blood volume. Thus, the consequent increase in blood volume in the chest region due to hydrostatic pressure possibly induced the reduction in the sympathetic modulation. In case of temperature reduction, the thermoreceptors located in the skin, subcutaneous tissue and blood vessels are stimulated, increasing flow redirection to protect the vital organs. . $^{3,19}$

These findings agree in part with Buchheit et al., ${ }^{8}$ Al Haddad et al. ${ }^{2}$, Stanley et al. ${ }^{25}$ and Parouty et al. ${ }^{21}$ who also noted faster HRV recovery using CWI, but over a shorter duration. Although the time of immersion was less than the $15 \mathrm{~min}$, the temperature of $14{ }^{\circ} \mathrm{C}$ was used in the above studies where CWI also promoted better results when compared to control condition. Al Haddad et al. ${ }^{2}$ emphasized that CWI performed at $14^{\circ} \mathrm{C}$ can stimulate cold receptors and promote a cumulative effect of hemodynamic responses through the thermoregulatory system, favoring better autonomic control.

Table 1

Mean and standard deviation of anthropometric variables and age.

\begin{tabular}{|c|c|c|c|c|c|c|}
\hline \multirow[b]{2}{*}{ Variables } & \multicolumn{6}{|l|}{ Groups } \\
\hline & $C G(n=20)$ & $\mathrm{G} 1(n=19)$ & $\mathrm{G} 2(n=19)$ & G3 $(n=18)$ & $\mathrm{G} 4(n=20)$ & $p$ value \\
\hline Age (years) & $21.48 \pm 2.64$ & $21.56 \pm 3.42$ & $22.19 \pm 3.15$ & $20.73 \pm 2.49$ & $22.00 \pm 2.85$ & 0.557 \\
\hline Weight (kg) & $76.60 \pm 13.41$ & $71.32 \pm 9.84$ & $73.86 \pm 9.46$ & $71.62 \pm 6.69$ & $75.50 \pm 11.17$ & 0.845 \\
\hline Height (m) & $1.74 \pm 0.06$ & $1.75 \pm 0.07$ & $1.75 \pm 0.06$ & $1.77 \pm 0.07$ & $1.77 \pm 0.07$ & 0.363 \\
\hline BMI $\left(\mathrm{kg} / \mathrm{m}^{2}\right)$ & $25.19 \pm 4.08$ & $24.08 \pm 3.60$ & $24.23 \pm 2.61$ & $23.04 \pm 2.35$ & $24.09 \pm 2.57$ & 0.460 \\
\hline
\end{tabular}

CG: control group; G1: $5^{\prime}$ at $9 \pm 1^{\circ} \mathrm{C}$; $\mathbf{G 2}$ : $5^{\prime}$ at $14 \pm 1^{\circ} \mathrm{C}$; $\mathbf{G 3}: 15^{\prime}$ at $9 \pm 1{ }^{\circ} \mathrm{C}$; $\mathbf{G 4 :} 15^{\prime}$ at $14 \pm 1{ }^{\circ} \mathrm{C}$. 

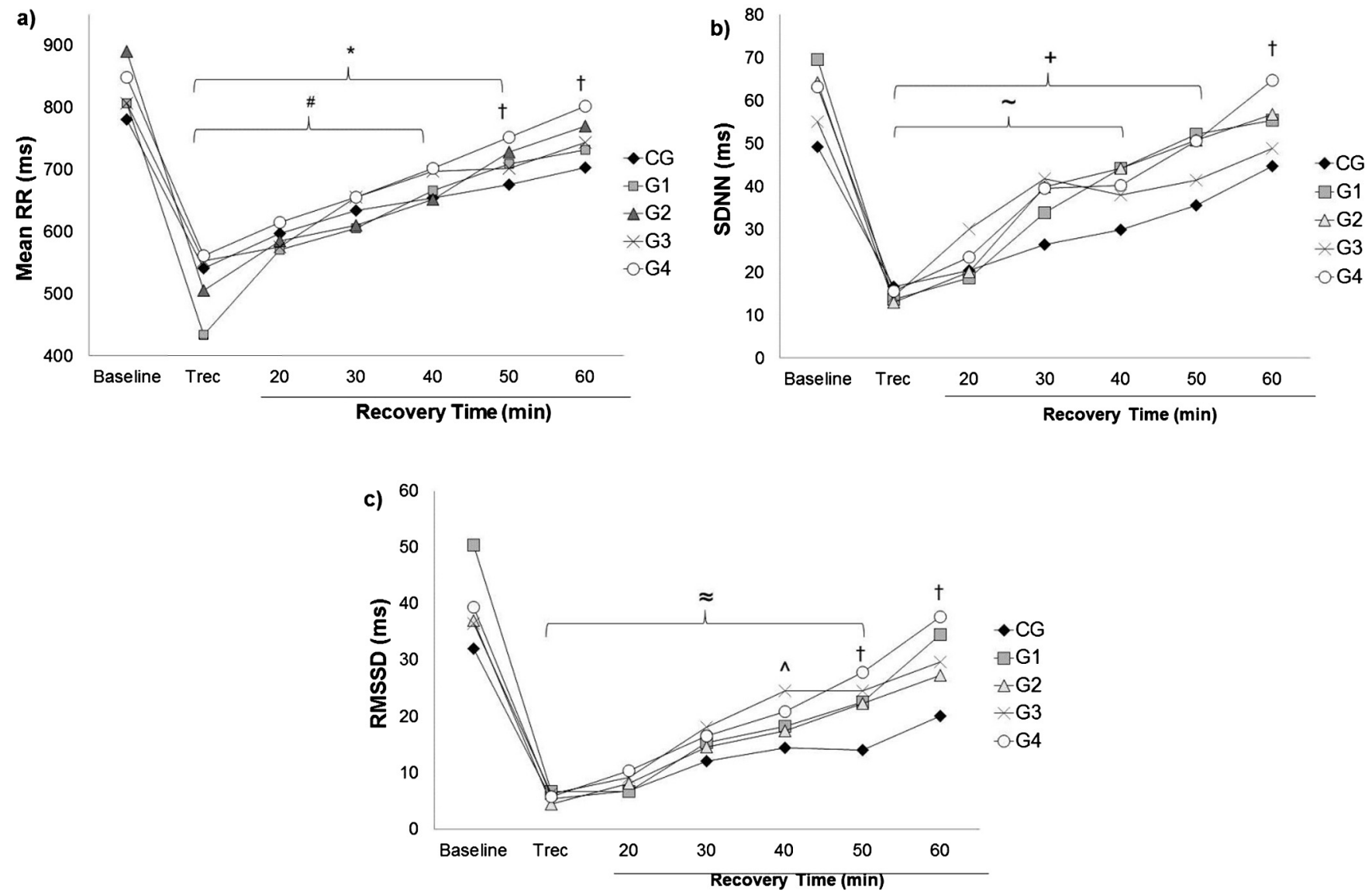

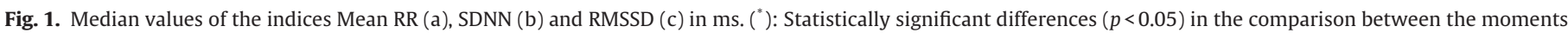

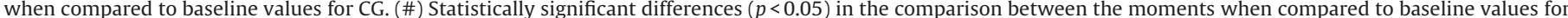

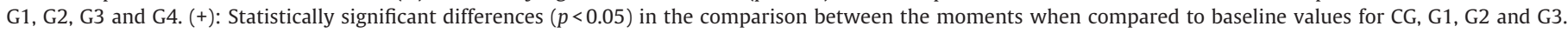

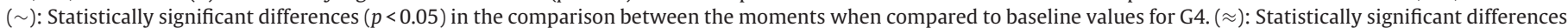

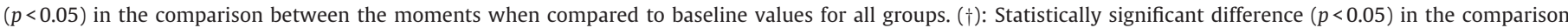

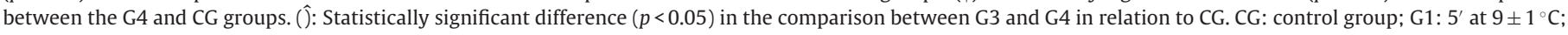
G2: $5^{\prime}$ at $14 \pm 1^{\circ} \mathrm{C}$; G3: $15^{\prime}$ at $9 \pm 1^{\circ} \mathrm{C}$; G4: $15^{\prime}$ at $14 \pm 1^{\circ} \mathrm{C}$.

The present study indicates that temperature and duration of immersion can be determining factors to optimize ANS response. Speculations about possible stress caused by immediate contact with CWI could justify the smaller effect on the recovery of G1 and G3 compared to the other groups. ${ }^{14}$ However, in VLF better recovery was observed in $\mathrm{G} 1$ compared to the control condition. It is known that VLF suffers, among other systems, influence of the thermoregulatory system, ${ }^{26,27}$ which due to lower temperatures may have promoted greater stimulation of thermoreceptors due to the sharp decrease in temperature after immersion ${ }^{2,8,25}$ even if observed for a short duration (5 min).

From a physiological point of view, the thermoregulation process is related to autonomic activity. Although this process is well established, detailed descriptions of the vagal afferents, its exact location and morphological type are uncertain. ${ }^{6}$ Schlader et al. ${ }^{24}$ corroborates these statements but describes the direct relationship between sensation/thermal comfort and behavior of the thermoregulatory system. In this case, the skin temperature has an important role considering the stimulation of its receptors with consequent thermal response. However, just as nerve conduction velocity $^{1}$, the answers of ANS after small temperature variations are unknown.

Based on the statements described, it can be inferred about the possibility of different responses on changes in technical application doses. The findings of this study reinforce the need for investigations aimed at a better understanding of the thermoregulation process and its relationship with the ANS.

Another aspect that also should be discussed concerns the physiological deleterious effects of CWI. Datta and Tiptom ${ }^{10}$ describe the mechanism of "cold-shock" as probable disturbing homeostasis, reflecting in cardiovascular risks. This condition, however, did not happen in the autonomic scope even in temperatures of $9^{\circ} \mathrm{C}$. Thus, all groups tested in this trial responded within safe limits at least on the basis of HRV.

Therefore, if the objective is cardiac autonomic modulation restoration, it can be inferred that the best application strategy of CWI is $15 \mathrm{~min}$ at $14^{\circ} \mathrm{C}$. Such strategies can promote an anticipated recovery in HRV indices, and are easily reproducible due to the lower thermal discomfort that, maintaining temperatures at around $14{ }^{\circ} \mathrm{C}$ can promote compared to lower temperatures often used in sports and scientific fields.

One limitation is worth mentioning. Although all participants were classified as physically active, according to IPAQ, it was impossible to ensure the specificity of the activities that the participants performed, and performance capacity was also not controlled, which constitutes a limitation, as the level and type of physical activity influence ANS. ${ }^{17}$ Specifically for this study, no statistical differences in baseline and during the application of CWI ensure that at least the same response profile for the primary variable HRV. To ensure more complete participants descriptions to the exercise 

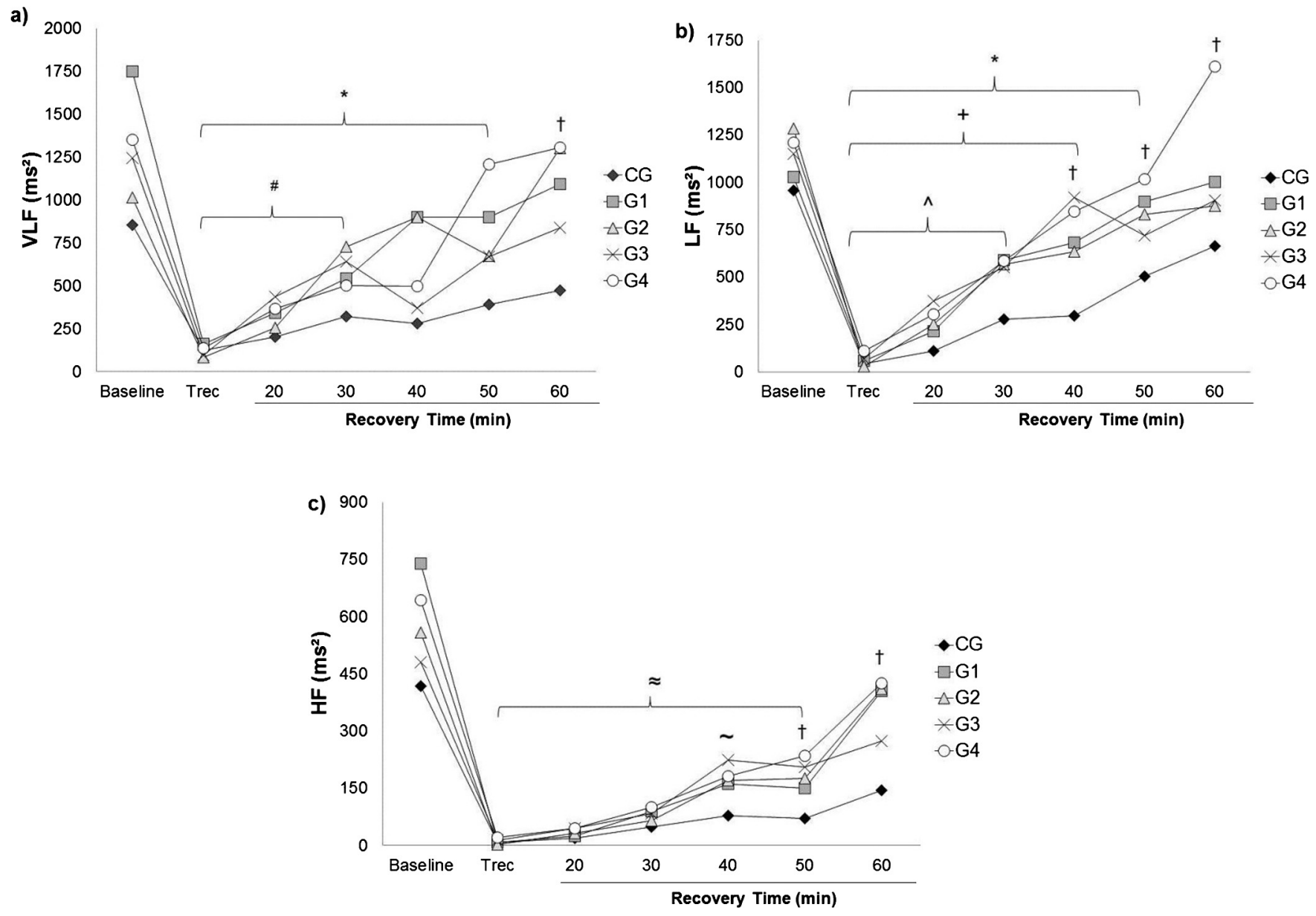

Fig. 2. Median values of VLF indices (a), LF (b) and $\mathrm{HF}(\mathrm{c})$ in $\mathrm{ms}^{2}$. ( $\left.{ }^{*}\right)$ : Statistically significant differences $(p<0.05)$ in the comparison between the moments when compared to baseline values for CG. (\#): Statistically significant differences $(p<0.05)$ in the comparison between the moments when compared to baseline values for G1, G2, G3 and G4. (+): Statistically significant differences $(p<0.05)$ in the comparison between the moments when compared to baseline values for G1 and G2. $(\hat{)})$ Statistically significant differences $(p<0.05)$ in the comparison between the moments when compared to baseline values for G3 and G4. $(\approx)$ Statistically significant differences $(p<0.05)$ in the comparison between the moments when compared to baseline values for all groups. ( $\dagger$ ): Statistically significant difference $(p<0.05)$ in the comparison between the G4 and CG groups. ( $\sim)$ Statistically significant difference $(p<0.05)$ in the comparison between the G3 and CG groups.CG: control group; G1: $5^{\prime}$ at $9 \pm 1{ }^{\circ} \mathrm{C} ; \mathrm{G} 2: 5^{\prime}$ at $14 \pm 1^{\circ} \mathrm{C}$; G3: 15 at $9 \pm 1{ }^{\circ} \mathrm{C} ; \mathrm{G} 4: 15$ 'at $14 \pm 1{ }^{\circ} \mathrm{C}$

a)

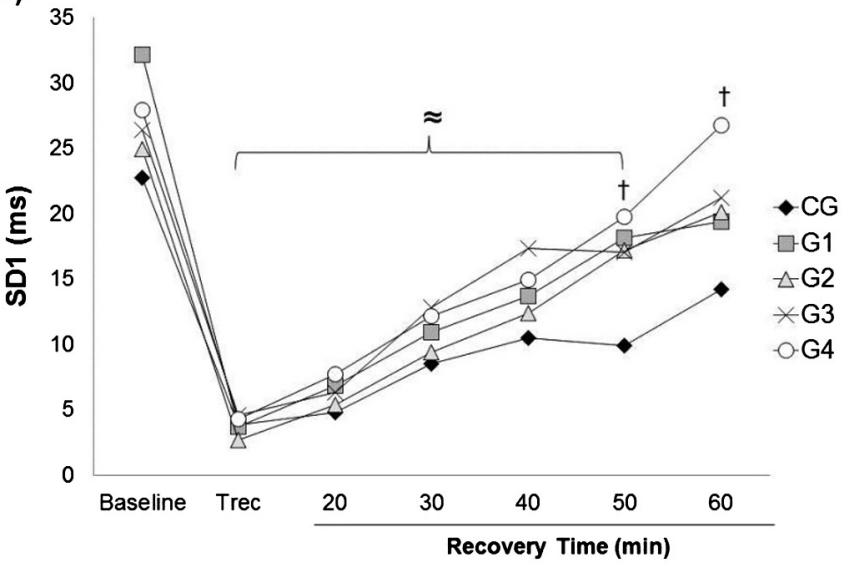

b)

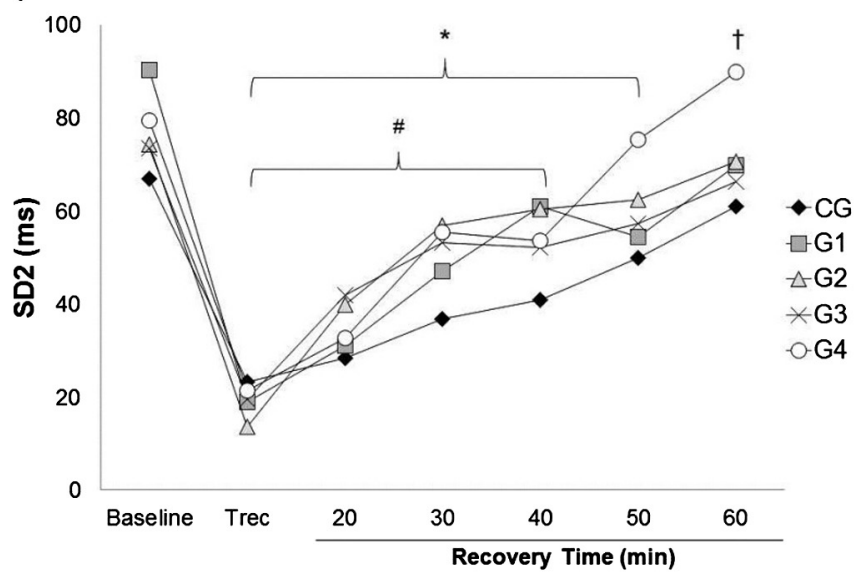

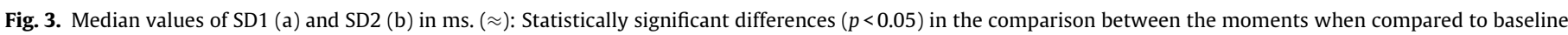

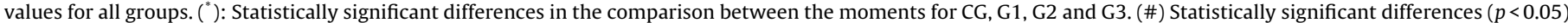

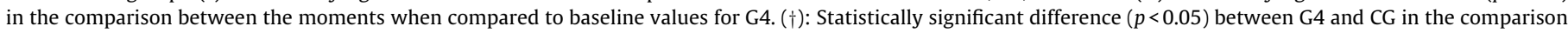
between groups. CG: control group; G1: $5^{\prime}$ at $9 \pm 1{ }^{\circ} \mathrm{C}$; G2: $5^{\prime}$ at $14 \pm 1^{\circ} \mathrm{C}$; G3: $15^{\prime}$ at $9 \pm 1{ }^{\circ} \mathrm{C}$; G4: $15^{\prime}$ at $14 \pm 1{ }^{\circ} \mathrm{C}$. 
profile, it's suggested, in future studies, the control of performance during the exercise protocol.

Thus, future studies are necessary addressing the duration and temperature application of CWI on other variables, such as clinical, biochemical and functional, in addition to considering the stress models and population investigated. Extrapolation of findings on CWI without considering such factors could promote bias in interpretation by readers who use science as means of investigating performance on a practical level in the field.

\section{Conclusion}

This study demonstrated the positive effects of CWI on the restoration of cardiac autonomic modulation, implying that CWI applied for $15 \mathrm{~min}$ at $14{ }^{\circ} \mathrm{C}$ is the most effective dosage for this purpose. It is worth noting that the period of $60 \mathrm{~min}$ post-exercise was sufficient to restore the values of the indices to pre-exercise conditions in physically active subjects, regardless of the technique applied.

\section{Practical implications}

The results of this study provide important information for clinical practice in sports medicine and exercise, considering the post-exercise recovery, suggesting that:

(1) Sixty minutes is enough for physically active people to recover their autonomic functions after high-intensity exercise.

(2) CWI can be indicated to accelerate the cardiac autonomic recovery.

(3) CWI best strategy considering temperature and duration is $15 \mathrm{~min}$ at $14^{\circ} \mathrm{C}$.

\section{Acknowledgments}

The authors would like to thank the CNPq (National Council for Scientific and Technological Development), CAPES (National Council for the Improvement of Higher Education) and FAPESP (São Paulo Research Foundation-number 13/12474-7) for financial support.

\section{References}

1. Algafly AA, George KP. The effect of cryotherapy on nerve conduction velocity, pain threshold and pain tolerance. Br J Sports Med 2007; 41(6):365-369.

2. Al Haddad H, Laursen PB, Chollet D et al. Effect of cold or thermoneutral water immersion on post-exercise heart rate recovery and heart rate variability indices. Auton Neurosci 2010; 156:111-116.

3. Al Haddad H, Laursen PB, Ahmaidi S et al. Influence of cold water face immersion on post-exercise parasympathetic reactivation. Eur J Appl Physiol 2010; 108:599-606.

4. Ascensão A, Leite M, Rebelo AN et al. Effects of cold water immersion on the recovery of physical performance and muscle damage following a one-off soccer match. J Sports Sci 2011; 29:217-225.

5. Bastos FN, Vanderlei LCM, Nakamura FY et al. Effects of cold water immersion and active recovery on post-exercise heart rate variability. Int J Sports Med 2012; 33(11):873-879.

6. Berthoud HR, Neuhuber WL. Functional and chemical anatomy of the afferent vagal system. Auton Neurosci 2000; 85(1-3):1-17.
7. Bleakley C, McDonough S, Gardner E et al. Cold-water immersion (cryotherapy) for preventing and treating muscle soreness after exercise. Cochrane Database Syst Rev 2012;(2):CD0082622 [Review].

8. Buchheit M, Peiffer JJ, Abbiss CR et al. Effect of cold water immersion on postexercise parasympathetic reactivation. Am J Physiol Heart Circ Physiol 2009; 296(2):421-427.

9. Buchheit M, Simpson MB, Al Haddad $\mathrm{H}$ et al. Monitoring changes in physical performance with heart rate measures in young soccer players. Eur J Appl Physiol 2012; 112(2):711-723.

10. Datta A, Tipton M. Respiratory responses to cold water immersion: neural pathways, interactions, and clinical consequences awake and asleep. J Appl Physiol 2006; 100(6):2057-2064.

11. Glasgow PD, Ferris R, Bleakley CM. Cold water immersion in the management of delayed-onset muscle soreness: is dose important? A randomised controlled trial. Physical Therapy in Sport 2014; x:1-6.

12. Gois MO, Campoy FAS, Alves $\mathrm{T}$ et al. The influence of resistance exercise with emphasis on specific contractions (concentric vs. eccentric) on muscle strength and post-exercise autonomic modulation: a randomized clinical trial. Brazilian Journal of Physical Therapy 2014; 18(1):30-37.

13. Goulopoulou S, Heffernan KS, Fernhall B et al. Heart rate variability during recovery from a Wingate test in adolescent males. Med Sci Sports Exerc 2006; 38:875-881.

14. Halson SL, Quod MJ, Martin DT et al. Physiological responses to cold water immersion following cycling in the heat. International Journal Sports Physiology and Performance 2008; 3(3):331-346.

15. Hoshi RA, Pastre CM, Vanderlei LCM et al. Poincaré plot indexes of heart rate variability: relationships with other nonlinear variables. Auton Neurosci 2013; 177(2):271-274.

16. Kellmann M. Preventing overtraining in athletes in high-intensity sports and stress/recovery monitoring. Scand J Med Sci Sports 2010; 20(Suppl. 2): S95-S102.

17. Martinmaki K, Rusko H. Time-frequency analysis of heart rate variability during immediate recovery from low and high intensity exercise. Eur J Appl Physiol 2008; 102(3):353-360.

18. Millar PJ, Rakobowchuk M, McCartney N et al. Heart rate variability and nonlinear analysis of heart rate dynamics following single and multiple Wingate bouts. Applied Physiology, Nutrition and Metabolism 2009; 34:875-883.

19. Mourot L, Bouhaddi M, Gandelin E et al. Cardiovascular autonomic control during short-term thermoneutral and cool head-out immersion. Aviat Space Environ Med 2008; 79(1):14-20.

20. Ottone VO, Magalhães FC, Paula F et al. The effect of different water immersion temperatures on post exercise parasympathetic reactivation. PLoS One 2014; 9(12):e113730

21. Parouty J, Al Haddad H, Quod M et al. Effect of cold water immersion on 100-m sprint performance in well-trained swimmers. Eur J Appl Physiol 2010; 109(3):483-490.

22. Pastre CM, Bastos FN, Netto Junior J et al. Métodos de Recuperação Pós-exercício: uma Revisão Sistemática. Rev Bras Med Esporte 2009; 15(2):138-144.

23. Rowsell GJ, Coutts AJ, Reaburn P et al. Effect of post-match cold water immersion on subsequent match running performance in junior soccer players during tournament play. J Sports Sci 2011; 29:1-6.

24. Schlader ZJ, Stannard SR, Mündel T. Human thermoregulatory behavior during rest and exercise-a prospective review. Physiol Behav 2010; 99(3): 269-275.

25. Stanley J, Buchheit M, Peake JM. The effect of post-exercise hydrotherapy on subsequent exercise performance and heart rate variability. Eur J Appl Physiol 2012; 112(3):951-961.

26. Tarvainen MP, Niskanen JP, Lipponen JA et al. Kubios HRV-heart rate variability analysis software. Comput Methods Programs Biomed 2014; 113(1):210-220.

27. Tripathi KK. Very low frequency oscillations in the power spectra of heart rate variability during dry supine immersion and exposure to non-hypoxic hypobaria. Physiol Meas 2011; 32:717-729.

28. Twist C, Eston R. The effects of exercise-induced muscle damage on maximal intensity intermittent exercise performance. Eur J Appl Physiol 2005; 94:652-658.

29. Vanderlei LCM, Pastre CM, Hoshi RA et al. Noções básicas de variabilidade da frequência cardíaca e sua aplicabilidade clínica. Rev Bras Cir Cardiovasc 2009; 24(2):205-217.

30. Versey NG, Halson SL, Dawson BT. Water immersion recovery for athletes: effect on exercise performance and practical recommendations. Sports Med 2013; 43(11):1101-1130. 